\title{
Information space is action space: perceiving the partial lengths of rods rotated on an axle
}

\author{
Claire F. Michaels $\cdot$ Robert W. Isenhower
}

Published online: 16 November 2010

(C) Psychonomic Society, Inc. 2010

\begin{abstract}
In a single experiment, perceivers held unseen rods at some position along their lengths and reported the two partial lengths - to the left and to the right of the hand. Wielding was mechanically limited to a vertical plane. Previous research suggested that the information exploited for this task is captured in a space created from the moment of inertia and gravitational torque. The experiment reported here attempted to replicate the relevance of that space and to ask how exploration might access it. Perceivers were given feedback on accuracy on Blocks 2 and 3 of a fourblock experiment, and their performance and position in information space were monitored. Exploratory movements were recorded. Judgments were shown to depend on inertial and gravitational torques, as expected. Analysis of exploratory movements suggested that occupying a locus in information space is equivalent to exploring at some angular acceleration. The apparent weighting of cues (gravitational and inertial torque), which might be interpreted as a cognitive process, was instead interpreted as a consequence of manner of exploration.
\end{abstract}

Keywords Haptics · Kinesthesis · Dynamic touch ·

Perceptual learning $\cdot$ Cue integration

One claim of Jacobs and Michaels's (2007) ecologically motivated theory of direct learning is that the information that might underwrite perception in some task exists on a low-dimensional manifold. At any time, a perceiver or

The research reported here was supported by the National Science Foundation under Grant BCS-0820154.

C. F. Michaels $(\square) \cdot R$. W. Isenhower

Center for the Ecological Study of Perception and Action,

Department of Psychology, U-20, University of Connecticut,

Storrs, CT 06269, USA

e-mail: Claire.Michaels@Uconn.edu perceiver-actor uses some information variable, which is represented as a locus on the manifold; learning, in turn, entails moving on the manifold toward the optimal locus for that task. Movement from one locus to another is hypothesized to be an information-guided process, rather than a search process. The dynamic touch paradigm has recently been employed as a test bed for this theory (Arzamarski, Isenhower, Kay, Turvey, \& Michaels, 2010; Jacobs, Silva, \& Calvo, 2009; Michaels, Arzamarski, Isenhower, \& Jacobs, 2008; Michaels \& Isenhower, in press). This research has demonstrated (1) that a one-dimensional space of inertial moments captures the variability in perceivers' judgments of object length (Michaels et al.) and width (Arzamarski et al.), (2) that when given feedback, perceivers move through the space toward the optimal for that property, and (3) that the locus in the space that a perceiver occupies is related to the how the object is wielded (Arzamarski et al.).

While we have characterized spaces of inertial variables as "information spaces" (e.g., by Michaels et al., 2008), they fall short of identifying the detectable properties of stimulation that might inform judgments or actions. An inertial variable that captures the variance in judgments or actions is better thought of as a distal stimulus, not a proximal stimulus. This remark applies equally to other researchers' characterizations of individual variables (e.g., moment of inertia, static moment, or mass) as "the information" for dynamic touch. The problem of identifying how the distal property is specified by detectable variables remains. The goal of the current contribution is to use what we know about important inertial variables to help identify an information space and to ask further how loci in that space are accessed.

A guiding intuition of the direct-perception approach to dynamic touch is that perceiving some property entails 
wielding the object so as to access information that specifies the to-be-perceived property (Riley, Wagman, Santana, Carello, \& Turvey, 2002; Turvey, Carello, \& Kim, 1990). This intuition, formalized as the co-specificity hypothesis, holds that the intention to perceive some property, the exploration engaged in, and the information exploited are mutually entailed. The exploration-information relation relates, in turn, to wellknown concepts in the Gibsonian approach to perception: An invariant is a property that remains unchanged over some transformation. Exploring appropriately is wielding so as to allow invariants to emerge: to make variants vary and invariants stay the same. Adopting the direct-learning approach (Jacobs \& Michaels, 2007), Arzamarski et al. (2010) extended the concept of co-specificity to learning: Learning what invariant to detect is equivalent to learning what transformation, what type of wielding, is needed. For example, to reveal information about moment of inertia on some axis (resistance to rotational acceleration on that axis) entails rotationally accelerating the object. Research has supported these hypotheses, showing that exploration differs depending on the property to be perceived and that learning is accompanied by changes in exploration (Arzamarski et al.). Such intimacies of perception and exploration have also been noted in other haptic subsystems (Drewing \& Ernst, 2006; Lederman \& Klatzky, 1987, 1990). There is reason to believe, then, that the information space of dynamic touch has a dual exploration space.

In this article, we apply the exploration- and informationspace approach to the perception of partial length. In the partial length paradigm, a perceiver holds a rod somewhere along its length and reports the length that the rod extends on one or both sides of the hand. Michaels and Isenhower (in press) demonstrated that a variable space derived from the first and third principal moments of inertia $\left(I_{1}\right.$ and $\left.I_{3}\right)$ by Michaels et al. (2008) failed to account for all partial-length perception. In particular, they found that if the object whose length is to be perceived is mechanically constrained to rotate in a vertical plane, gravitational torque contributes to the information space. In the case of free wielding, too, the $I_{1} I_{3}$ space was insufficient, and either gravitational torque or "off-diagonal" terms from the inertia tensor contributed to the space (see also Carello, Santana, \& Burton, 1996). These conclusions were drawn from regression analyses of judgments against the mechanical variables.

In the research reported here, we ask how exploration makes information available and how changes in the exploitation of information are to be understood. We know already that whether perceivers are to report length or width affects how they explore (Arzamarski et al., 2010; Riley et al., 2002). A further demonstration that location in information space of dynamic touch reflects exploration would not only illustrate J. J. Gibson's (1966) concept of obtained (vs. imposed) information, but, more important, could help distinguish between the theory of direct perception (and direct learning) and cue-combination approaches. Cue-combination approaches include Bayesian, Brunswikian, and connectionist learning, which, for present purposes, we lump together. In what follows, we elaborate the similarities and differences between the ecological and combinatorial views in the context of Michaels and Isenhower's (in press) findings on wielding constrained to a vertical plane. Attention is then turned to an experiment that tests whether changes in manners of exploration can distinguish these two general approaches.

A key requirement of a hypothesized information space for some task is that it includes all variables that perceivers use to accomplish the task. As a starting point, we have often begun with seemingly elementary variables. For example, Michaels et al. (2008) used two principal moments of inertia, $I_{1}$ and $I_{3}$, which previous research had suggested were relevant, to create a one-dimensional inertial space for length perception in dynamic touch. (This inertial space would presumably map onto a corresponding information space.) A space created from two variables is one- rather than two-dimensional; the locus in the space describes the relative weighting of the two variables. The coefficients of a multiple regression of judgments against the two variables can locate the learner in the space, and also, therefore, track the learner's progress over trials.

In the direct learning theory, the information space is primary, and the variables used to construct it are incidental. It is even expected that an equivalent space could be constructed from other, related variables. All loci in the space are seen as having the same status; none are seen as more elementary, and none are seen as mixtures. An individual locus in the space is thereby viewed as an address, not as the recipe of a mixture. This pronouncement does not, of course, make it so, and those that promote a combinatorial view have a different pronouncement: Variables (usually called cues) are combined according to recipes based on past experience, and they, too, can be captured with multiple regression. Here, then, is the problem: Is it possible to distinguish two systems, one that accesses an information locus directly and a second that detects elementary properties and engages in some combinatorial calculation? The latter view is a legacy of Helmholtzian theory that endures in all modern cognitive approaches to perception. Bayesian theory has several versions of how to compute the likelihood functions of cue combinations (see Yuille \& Bülthoff, 1996, for an enumeration). Brunswik's lens model has perceivers setting appropriate weights for cues (e.g., Brunswik, 1952). Connectionist networks achieve appropriate weightings of input variables through interactive dynamics.

As intimated earlier, our working assumption is that a style of wielding a rod directly accesses a locus in the 
space. The corollary of this is that even though certain mathematical and statistical operations (e.g., multiple regression) can identify putative parts assembled by algorithm, these parts and algorithms need not have any psychological reality. In other words, we deny that cues are combined. Drewing and colleagues (Drewing \& Ernst, 2006; Kaim \& Drewing, 2010) have made an analogous argument for haptic exploration, asserting that the modulation of exploratory force functionally weights the cues of position and force in haptic shape perception.

Let us see how "direct access" might work for partial length perception of a rod fixed on an axle such that it can be rotated only in a vertical plane (Michaels \& Isenhower, in press). Perceivers were able to report the two partial lengths successfully. ${ }^{1}$ Michaels and Isenhower found that a onedimensional variable space derived from moment of inertia and gravitational torque explained the systematic variance in perceivers' judgments. The research reported here takes this general finding as its departure point. The ability to report the partial lengths of a rod rotated on an axis is a convenient starting point for establishing an information space and for investigating the information-exploration relation because the possible physical basis is limited to the moment of inertia, $I$, and gravitational torque, $T_{g}$. The muscular torque, $T_{m}$, needed to accelerate the rod some amount is the sum of the inertial and gravitational torques.

Let us review the variable space identified by Michaels and Isenhower (in press). The space was constructed from $I$ and $T_{g}$. Michaels and Isenhower assumed that the variables populating the space are sums of different weightings of $I$ and $T_{g}$. A locus on the space was identified by the ratio of these weightings. To assess the usefulness of different loci for the perception of partial length, Michaels and Isenhower regressed actual partial lengths against $a \bullet I+T_{g}$, for integer values of $a$ from 0 to 1,000 . The values of $I$ and $T_{g}$ were from stimulus objects used in that study and in the present one. The result, which is plotted in Fig. 1, can be read as follows: If a perceiver weighted $I$ relative to $T_{g}$ by the factor $a$, judgments and actual lengths could have correlations as high as the curve at that point. When $a=0$, performance (the correlation of actual and perceived partial length) could be no higher than .63 , the correlation between $T_{g}$ and actual length. When $a$ is large and $T_{g}$ is swamped by $I$, performance would similarly be limited to about .83 , the correlation between actual length and $I$. The optimal is 46 $\left(10^{1.66}\right.$ from Fig. 1), which is, of course, the ratio of the coefficients of $I$ and $T_{g}$ for the multiple regression of actual length against $I$ and $T_{g}$.

\footnotetext{
${ }^{1}$ As an aside, perceivers' success in the fixed plane was by itself surprising because accuracy in partial length perception is usually explained, in part, with reference to an off-diagonal term in the inertia tensor (Carello et al., 1996), which was unavailable with wielding constrained to a single plane.
}

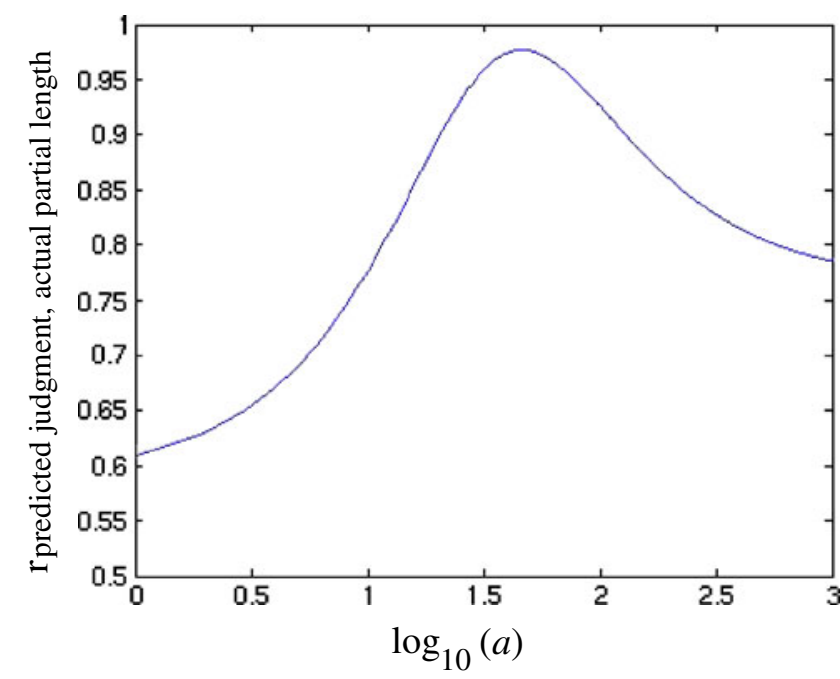

Fig. 1 The usefulness of different variables (loci on the information line) for specifying the partial length of a rod rotating on a fixed axis in a vertical plane. Usefulness was measured by correlations between predicted judgments $\left(\widehat{J}=a \cdot I+T_{g}\right)$ and actual lengths for different loci (values of $a$ ), which ranged from 0 to 1,000

Information use and exploration The idea that loci in a variable space might be self-standing variables, rather than combinations, can now be illustrated for the $I-T_{g}$ space. The locus 0 on the line, just because it happens to equal $T_{g}$, is not of a different kind from a locus of $a=1$, which can be computed as the simple sum of $I$ and $T_{g}$. In a cuecombination view, $I$ and $T_{g}$ are two cues that are detected independently and combined by a cognitive process. In the direct view, there is what might be called specific exploration: Detecting different loci entails different exploratory patterns. As intimated above, the hypothesized specificity of exploration provides a means by which cue combination and information detection can be distinguished experimentally.

A review of rotational dynamics quickly reveals how the "variable space" becomes and information space, and how exploration could access particular loci on the resulting information line, and thereby functionally "weight cues." As Newton's second law applies to rotational accelerations, the muscle torque $\left(T_{m}\right)$ needed from the wielder is a function both of the gravitational torque and the inertial torque - the product of the rod's moment of inertia and its angular acceleration. For a rod with unequal partial lengths, $T_{g}$ aids in rotating the shorter side up, but resists rotating the longer side up. In Equation 1, $m$ is rod mass; $g$ is the acceleration due to gravity; $\alpha$ is the angle of the rod from the horizontal; $b$ is the distance from the rotation point to the rod's center of mass; and $\ddot{\alpha}$ is the rotational acceleration.

$T_{m}=\ddot{\alpha} \cdot I+\cos (\alpha) \cdot b \cdot m \cdot g$

In the partial-length paradigm, most of the variables in Equation 1 are not under the control of an experimental participant (viz., $I, b, m$, and $g$ ). What a participant can 
manipulate in wielding are the rod's orientation and angular acceleration. Equation 1 reveals the solutions to how to mechanically weight $I$ and $T_{g}$. Accelerating the rod by some fixed amount weights $I$ by that amount. Thus, learning to optimally explore a collection of rods for partial length might mean learning to accelerate the rod at the alreadynoted optimum of $a$ (46), to which we can now add the units, radians $/ \mathrm{s}^{2}$ and establish dimensional homogeneity. Because we take $T_{m}$ to be a detectable variable, the identification of $a$ with $\ddot{\alpha}$ converts the space based in part on an object property $(I)$ into an information space. Moreover, the intended acceleration constitutes an action space. The action space ought to be equivalent to the information space created by regressing judgments against $I$ and $T_{g}$. Thus, a perceiver's locus in information space should resemble their locus in action space. Moreover, if learning to perceive partial length for these stimuli entails learning the appropriate acceleration, participants' movements in an acceleration space should resemble their movements in the information space.

Equation 1 suggests a second solution to how exploration might differentially weight $I$ and $T_{g}$. A wielder can manipulate the relative contribution of $T_{g}$ by changing the general orientation of the rod. If the rod were held vertically, for example, $T_{g}$ is 0 . As the orientation ranges from horizontal to near vertical, the acceleration needed to achieve specification decreases. In short, the onedimensional information space could correspond to a twodimensional action space, an alternative that movement data on explorations would easily reveal.

The goals of the experiment can now be made explicit: to replicate the vertical-plane findings of Michaels and Isenhower (in press), with two critical additions. First, we measure explorations to see whether there are relations between loci in information space and characteristics of exploration. Second, we ask whether training with feedback is accompanied by a movement in information space, as is expected from the theory of direct learning. If there is such movement, we can ask whether it, too, can be identified with changes in exploration, perhaps converging on the optimal acceleration of $46 \mathrm{rad} / \mathrm{s}^{2}$, or by modulating both acceleration and angle such that the net ratio is 46 .

\section{Method}

Participants Six students at the University of Connecticut participated in the experiment for partial course credit. All were right-handed.

Design Wielding was constrained to a single plane. Each participant was given 4 blocks of 21 trials. On the second and third blocks, feedback on accuracy was given; the first and last blocks were a pretest and a posttest. On all trials, the participant's wielding movements were recorded.

Apparatus Michaels and Isenhower's (in press) five wooden dowels, each $1.2 \mathrm{~cm}$ in diameter, were used in the stimulus set. Small holes drilled at various locations along each dowel served as the axis of rotation for each of the stimuli (see Table 1 for details of the objects). Participants wielded each of the five dowels around each of its rotation points, creating a total of 21 stimulus objects. Plasterboard separated the participant from the to-be-perceived objects. The participant's right arm rested on an armrest, and the hand extended through a hole in the plasterboard, which was covered by a flap of dark cloth, occluding view (see Fig. 2). Centered above the hole, a meter stick was affixed to the board.

The rod was mounted on a fixed metal pin by inserting the pin into one of the holes in the rod. The pin constrained the movement of the rod to rotation about a single axis. The participant grasped the rod at the location of the pin. Hand orientation was sampled at $120 \mathrm{~Hz}$ using a magnetic tracking system (Polhemus Fastrak, Polhemus Corporation, Colchester, VT) and 6-D Research System software (Skill Technologies, Inc., Phoenix, AZ). The marker was taped to the back of the participant's hand as shown in Fig. 2.

Procedure The rod was placed on the axle, and the participant gripped the rod firmly with the right hand so that the axle was between the middle and ring fingers. The participant's forearm was lightly restrained to the armrest with Velcro to keep the arm centered relative to the length indicator. Participants made partial length judgments by verbally identifying the positions on the meter stick that corresponded to the perceived left and right ends of the rod. The 50-cm mark on the meter stick was directly above rotation point. On any one trial, the participant's hand was guided to grasp the horizontally oriented rod, and the marker was electronically aligned. Recording began, and the participant wielded the rod as long as desired. On test trials (i.e., in Blocks 1 and 4) recording terminated when the judgment was given; on feedback trials, the post-feedback wielding was also recorded, though those movements were not analyzed.

Feedback about the actual positions of the ends relative to the meter stick was given verbally after the participant gave their judgment. Participants were encouraged to again wield the rod after the feedback had been given.

\section{Results}

Performance The two perceived partial lengths were computed for each trial as the distance between the identified positions of the rod ends and the $50-\mathrm{cm}$ mark. 
Table 1 Partial lengths, whole lengths, and mechanical properties of stimulus objects
The value for torque is the gravitational torque for a horizontally oriented rod

\begin{tabular}{|c|c|c|c|c|c|}
\hline Left (cm) & Right $(\mathrm{cm})$ & Total $(\mathrm{cm})$ & Mass (g) & $I\left(\mathrm{~kg}-\mathrm{m}^{2}\right)$ & Torque $(n-m)$ \\
\hline 8.0 & 17.0 & 25 & 80 & .00058 & .0353 \\
\hline 12.5 & 12.5 & 25 & 80 & .00042 & .0000 \\
\hline 17.0 & 8.0 & 25 & 80 & .00058 & -.0353 \\
\hline 13.0 & 23.0 & 36 & 104 & .00138 & .0510 \\
\hline 18.0 & 18.0 & 36 & 104 & .00112 & .0000 \\
\hline 23.0 & 13.0 & 36 & 104 & .00138 & -.0510 \\
\hline 20.5 & 24.5 & 45 & 144 & .00250 & .0283 \\
\hline 22.5 & 22.5 & 45 & 144 & .00244 & .0000 \\
\hline 24.5 & 20.5 & 45 & 144 & .00250 & -.0283 \\
\hline 15.0 & 30.0 & 45 & 144 & .00325 & .1062 \\
\hline 30.0 & 15.0 & 45 & 144 & .00325 & -.1062 \\
\hline 21.0 & 32.0 & 53 & 152 & .00402 & .0819 \\
\hline 26.5 & 26.5 & 53 & 152 & .00356 & .0000 \\
\hline 32.0 & 21.0 & 53 & 152 & .00402 & -.0819 \\
\hline 18.5 & 34.5 & 53 & 152 & .00453 & .1192 \\
\hline 34.5 & 18.5 & 53 & 152 & .00453 & -.1192 \\
\hline 28.0 & 34.0 & 62 & 177 & .00583 & .0520 \\
\hline 31.0 & 31.0 & 62 & 177 & .00567 & .0000 \\
\hline 34.0 & 28.0 & 62 & 177 & .00583 & -.0520 \\
\hline 25.0 & 37.0 & 62 & 177 & .00631 & .1041 \\
\hline 37.0 & 25.0 & 62 & 177 & .00631 & -.1041 \\
\hline
\end{tabular}

Correlations between perceived and actual partial lengths were computed for individual participants on each block of trials. The results are plotted in Fig. 3. Fisher Ztransformations of these correlations were subjected to an analysis of variance (ANOVA) with repeated measures on block. The effect of block was not significant, $F(3,15)=$ $3.02, p=.065, \eta_{\mathrm{p}}^{2}=.376$, though omitting the single participant who performed the best on Block 1 and who did not improve over blocks elevated the $F$ to significance.

Although we weren't explicitly interested in calibrationthe metric agreement between actual and perceived lengthfor completeness we note that the average absolute error decreased over blocks of trials, $F(3,15)=20.79, p<.001$, $\eta_{\mathrm{p}}{ }^{2}=.806$. The average errors on successive blocks were

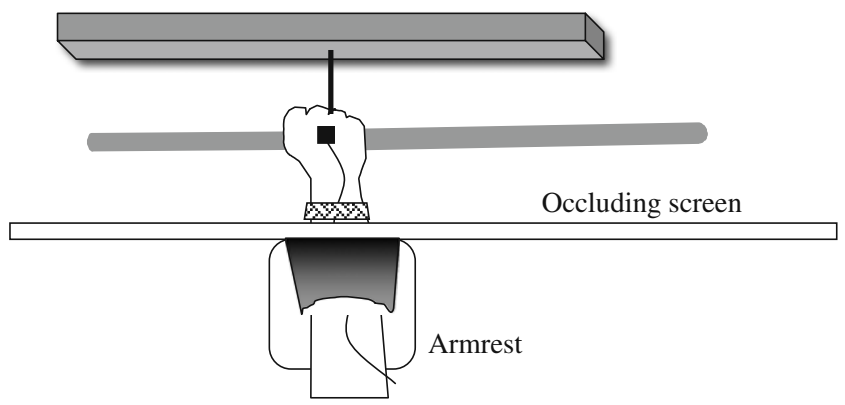

Fig. 2 A top view of the rod, axle, occluding screen, flap, and participant's hand. The Polhemus marker is shown on the back of the perceiver's hand. A band of Velcro loosely held the Polhemus wire in position
8.4, 5.0, 4.4, and $4.6 \mathrm{~cm}$, respectively. An analysis of constant error revealed that participants tended to underestimate length.

Information space To locate a perceiver in the $I-T_{g}$ variable space, we regressed perceived partial length against $I$ and $T_{g}$ (assuming a horizontally oriented rod) for each block of trials

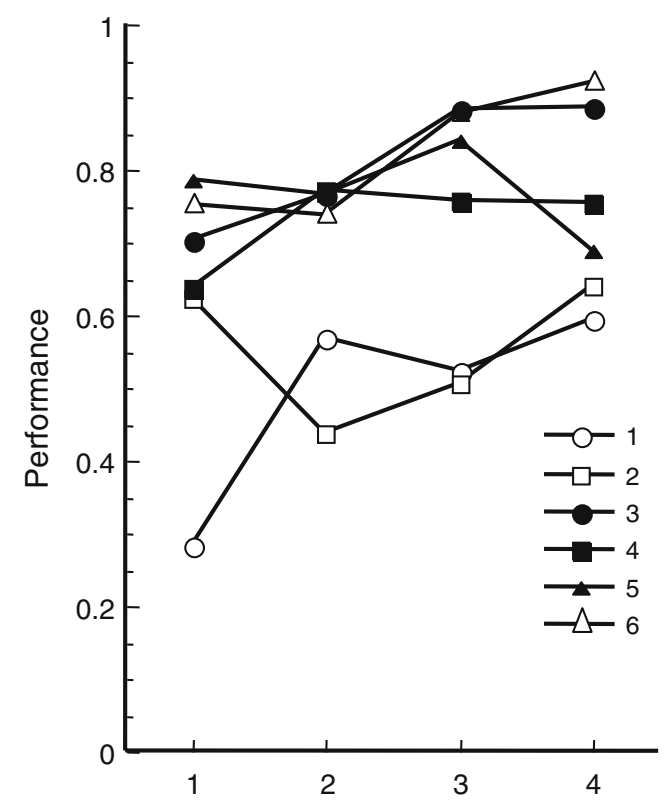

Fig. 3 Individual participants' correlations between perceived and actual partial lengths, given as a function of blocks 


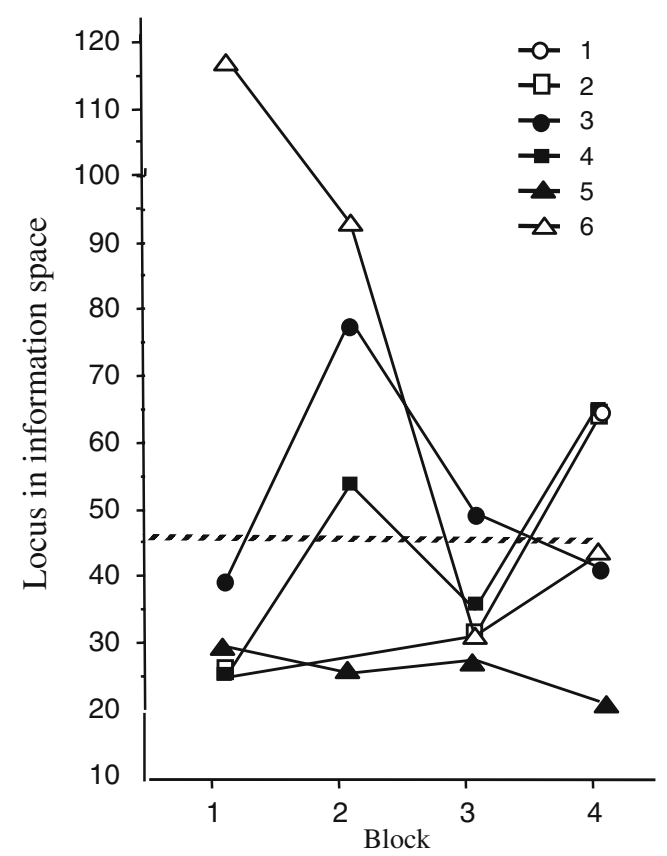

Fig. 4 The occupied loci in variable space over blocks of trials. The striped line represents the optimal locus. Missing points represent blocks on which $T_{g}$ did not achieve statistical significance

and calculated the ratio of the resulting coefficients, yielding the locus $a$, referred to earlier. The results are plotted by participants in Fig. 4. On 3 of the 24 block-by-participant cells, the contribution of $T_{g}$ was not significant, leaving the ratio undefined, so these blocks are not included in the figure. The average $R$ for the multiple regressions was .808 . This replicates Michaels and Isenhower's (in press) demonstration of the appropriateness of the $I-T_{g}$ variable space.

A perceiver is expected to start at some arbitrary point on the information line, and learning, if it occurs, should move the perceiver in the direction of the optimal. On Block 1, there was a substantial range of occupied loci, from well below the optimal of 46, to well above it. The changes from Block 1 to Block 2 were noisy, in part because of the general recalibration that occurs with the first few feedback trials. ${ }^{2}$ Feedback is also expected to yield consistent movement toward the optimum in the information space. This was not the case; over blocks with feedback, the majority demonstrated little apparent convergence on the optimum. In other words, the learning demonstrated in Fig. 3 depended more on reduced variability of judgments, than it did on reattunement. That only some participants showed reliable reattunement was a disappointment because it limited the degree of change that

\footnotetext{
$\overline{2}$ Average perceived length ranged from 11.9 to $20.0 \mathrm{~cm}$ over participants on Block 1. (The actual partial lengths averaged $25 \mathrm{~cm}$.) The first few trials of feedback recalibrate judgments; the Block 2 average perceived lengths ranged from 20.8 to 22.8 . These trials on which calibration can change dramatically, contribute substantial noise to the regression analyses.
}

we hoped to find paralleled in exploration. Nevertheless, the large individual differences are good fodder for examining relations between information and action spaces.

Exploration space Let us now examine the exploratory movements. We begin with descriptive measures, which included peak frequency of oscillation, duration of exploration, angular excursion peak in each direction, as well as peak acceleration on each trial. For acceleration, the rodangle time series was filtered with a low-pass Butterworth filter with a cutoff of $5 \mathrm{~Hz}$, and the second time-derivative of this signal was computed; we singled out a discrete measure of acceleration, the peak during the middle half of the exploration. ${ }^{3}$ Because individual differences were expected, ANOVAs on these dependent variables were done as participant by block designs. We tested various other additional independent variables (e.g., left vs. right side, shorter vs. equal vs. longer sides). In general, these analyses revealed that the biggest source of variation was participant. Given these individual differences, we present the various means in Table 2 only for descriptive purposes.

We also did a crude classification of the angleacceleration plots. The three most frequently observed categories, examples of which are plotted in Fig. 5, were highly repetitive linear relations $(60 \%$ of trials) and flattened (13.5\%) trajectories, and the more irregular "cascades" (15.5\%). The linear relation shows acceleration proportional to angular displacement, whereas the flattened indicates a softening spring, but only as the extreme of supination is reached. The remaining $11 \%$ were distributed over categories of discrete movement (in which rod acceleration went to 0 at one or both extremes), jiggling, and multi-stiffness linear plots, in which there were two or more linear clusters with different steepness. Some trials showed more than one category, but were classified according to which category dominated; the examples given in Fig. 5 are pure examples. Classifications were not evenly distributed over participants, nor over partial lengths (e.g., rotating the short end up vs. rotating the long end up). In short, the search for a single, optimal kinematic pattern onto which participants might converge came

\footnotetext{
${ }_{3}$ There were a number of discrete estimates of acceleration that might have been used: the maximal acceleration, the average of the per-cycle maxima, median of the maxima, etc. Obviously an acceleration peak might be an incidental consequence of a noisy torque. On the other hand, measures of the central tendency of acceleration peaks are also not necessarily the best option. Determining the point in the exploration that the wielder found what they were looking for is a challenge. A good guess might be that it happens near the end of the exploration. However, we found that average peak acceleration during the last third of a trial did not predict judgments better than average peak accelerations, which tended to be slightly better than the absolute peak accelerations per trial. Given the rough predictive equivalence of these various measures, we arbitrarily chose the peak acceleration in each direction during the middle half of the exploration.
} 
Table 2 General characteristics of exploration trials analyzed by participants

\begin{tabular}{|c|c|c|c|c|c|c|}
\hline \multirow[b]{2}{*}{ P. } & \multirow[b]{2}{*}{ Duration (s) } & \multirow[b]{2}{*}{ Frequency $(\mathrm{Hz})$} & \multicolumn{2}{|c|}{ Peak acceleration } & \multicolumn{2}{|c|}{ Peak angular excursion } \\
\hline & & & $\begin{array}{l}\text { Clockwise } \\
(\mathrm{rad} / \mathrm{s} / \mathrm{s})\end{array}$ & $\begin{array}{l}\text { Counterclockwise } \\
(\mathrm{rad} / \mathrm{s} / \mathrm{s})\end{array}$ & $\begin{array}{l}\text { Clockwise } \\
\text { (rad) }\end{array}$ & $\begin{array}{l}\text { Counterclockwise } \\
\text { (rad) }\end{array}$ \\
\hline 1 & 11.3 & .92 & 17.3 & 15.6 & .537 & .309 \\
\hline 2 & 17.4 & .61 & 30.5 & 24.7 & 1.341 & .508 \\
\hline 3 & 19.7 & 1.59 & 25.0 & 24.3 & .340 & .222 \\
\hline 4 & 12.0 & 1.16 & 44.9 & 44.1 & .669 & .391 \\
\hline 5 & 16.9 & .79 & 23.8 & 22.9 & .877 & .276 \\
\hline 6 & 9.9 & 1.31 & 84.5 & 68.4 & 1.601 & .607 \\
\hline $\bar{x}$ & 14.5 & 1.06 & 37.7 & 33.3 & .894 & .386 \\
\hline
\end{tabular}

quickly to a halt. Noteworthy is that all of the various patterns met with substantial success in revealing partial length.

Consistent kinematic details over participants, however, were not central to the hypothesis that the information space is an exploration space. That hypothesis requires only that $T_{m}$-the muscle torque needed to accelerate the rod at the rate identified by the information-line locus - is the information supporting partial length perception. Evidence for this hypothesis could come from demonstrations (1) that the block averages of accelerations observed during exploration would mirror the occupied loci in information space presented in Fig. 4 and (2) that exerted muscle torque would explain the variance in perceivers' judgments of partial length. 4

To determine whether occupied loci in information space were mirrored in acceleration space, we determined the peak angular acceleration on each direction on each trial, and then averaged them over directions and trials. The results are presented by participant and block in Fig. 6. We note first the individual differences in the first block, with average accelerations ranging from 10 to $90+\mathrm{rad} / \mathrm{s}^{2}$. For four of the participants, acceleration was fairly low, and changed little over blocks. For the other two participants, acceleration was higher, and changed in the direction of the optimal information space value identified earlier, 46.

There are similarities between the information space of Fig. 4 and the action space of Fig. 6. Participants maintained the same crude positions on the two graphs, and the two moving participants from Fig. 6 show roughly corresponding movements in Fig. 4. The correlation between the information-space loci and the action-space loci for all participants was a significant thought modest .62,

\footnotetext{
${ }^{4}$ The particular hypothesis that $T_{m}$ informs of length assumes that accelerations are roughly the same for different object lengths. This was indeed the case; the average accelerations for short, equal, and long sides of the le would be a $T_{m}$ space in which participants (learn to) exert a particular torque on all trials, and judgments would be informed by the resulting acceleration.
}

$p<.02$. We take this agreement as tentative support for our broad hypothesis that the information space for length perception in dynamic touch is an action space.

To test the corollary prediction that judgments are constrained by $T_{m}$, we computed $T_{m}$ for each trial using Equation 1, first with orientation set to horizontal, and using that trial's observed clockwise and counter-clockwise accelerations. Note that on each trial, two values of $T_{m}$ are relevant, one in the (participant's) clockwise direction, which informs about the length of the left side of the rod, and one in the counterclockwise direction, which informs about the right side. We computed how well each participant's 168 partial-length judgments correlated with the corresponding, computed $T_{m}$. The results are presented in the All column of Table 3. With the exception of Participant 1, who was also the poorest performer, the correlations are fairly high: Exerted muscle torque predicted judgments reasonably well. Note that this analysis ignores blocks; thus any learning simply adds to the noise. The All row shows the correlation of the data pooled over participants. The within-block correlations, which do allow for learning, were higher; their average is presented in the bottom row. All correlations were significant at the .001 level.

We made various attempts to include orientation in the information space, as invited by Equation 1 (e.g., the peak angular excursion and the angle at which peak acceleration occurred), but were uncertain as to what momentary orientation or range was important to the participant. Also, the various measures of orientation were correlated with acceleration (see Fig. 5), making it difficult to tease apart their contributions.

A possible reason that $T_{m}$ doesn't account for even more variance in judgments is related to active vs. eccentric muscle contractions in creating torque: The $T_{m}$ s needed to rotate a rod with unequal partial lengths obviously differ with direction. Rotating the longer side up requires not only torque to overcome rotational inertia, but also to overcome gravitational torque. There are two ways, however, to rotate the shorter side up. One could either let gravity do the 

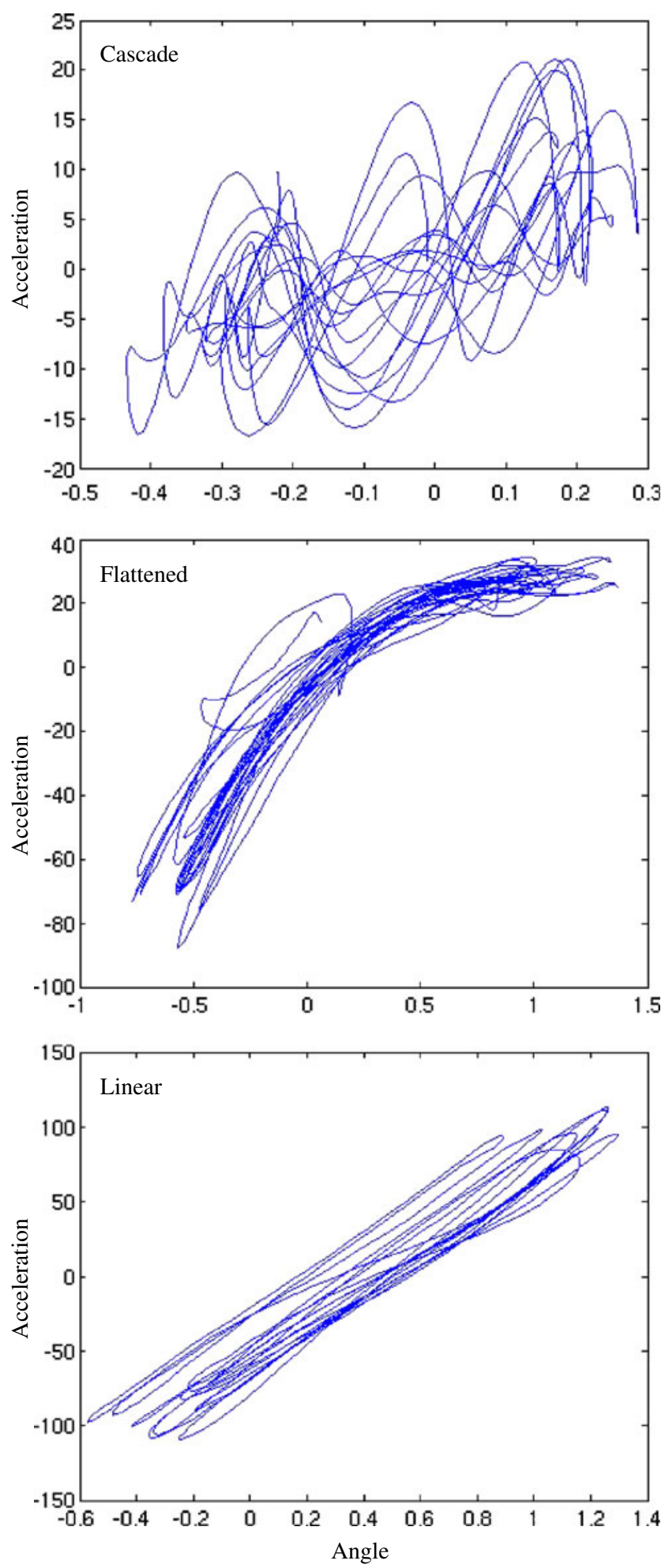

Fig. 5 Acceleration-angle plots illustrating the three most frequently observed categories of movements: cascades, flattened, and linear relations. Notice that the axes have different scales. An angle of zero is a horizontal rod orientation. Positive angles are supination; negative angles are pronation

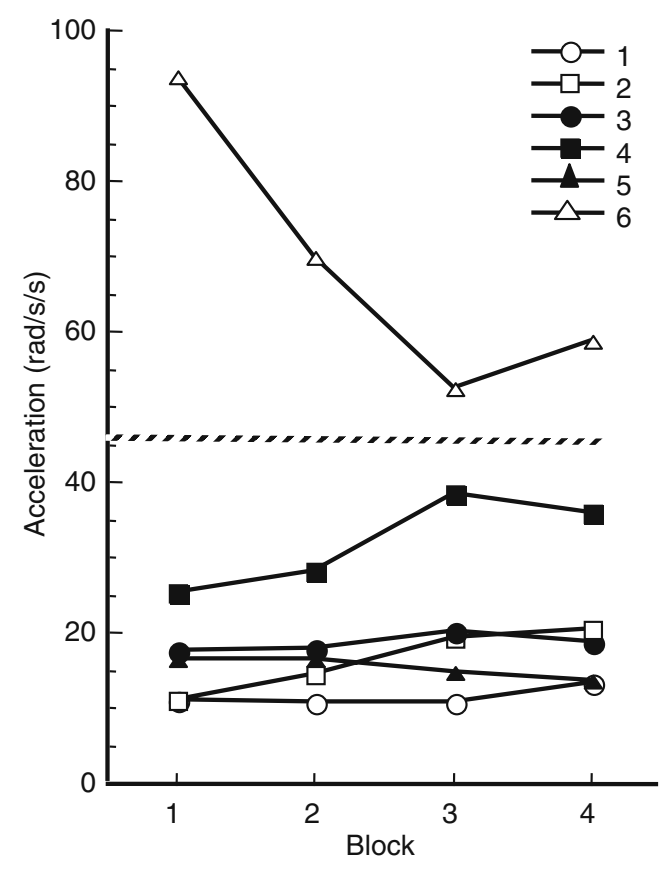

Fig. 6 Average peak observed angular acceleration in the Fixed Condition of Experiment 4, broken down by participant and block. The striped line is the optimal acceleration

work, or one could actively accelerate the rod beyond the rate that gravity would create. In the former case, one is not really using $T_{m}$ to accelerate. Trials on which wielders failed to go beyond the acceleration created by gravity alone might, therefore, be expected to show a low correlation between (the negative) $T_{m}$ and judgment. To test this, we compared the predictive power of $T_{m}$ when acceleration was greater than would be caused by gravity alone and when it was not; the results are also presented in Table 3. Acceleration of the short side did not overcome gravity on roughly one-quarter of the trials, though participants differed dramatically, with Participant 6 demonstrating only 2 such trials. When $T_{m}$ was greater than 0 , it was a strong predictor of judgments; when it was less than zero it did not predict judgments. The non-significant correlations of $T_{m}$ and judgment for the negative subset of data indicate the apparent necessity of positive torques. A fishing expedition to determine what variable did predict judgments in the negative-torque cases revealed that the judgments of 4 of the 5 participants for whom negative torques occurred were most highly correlated with the perceived length of the other side (last column of Table 3). It is also interesting to note that the numbers of these negative-torque trials remained constant over blocks; that is, participants did not learn to generate the higher torques needed to surpass the acceleration achieved from gravity alone and to benefit from the concomitant information on the length of the shorter side. 
Table 3 Correlations between judgments and muscle torques

Negative torques are when the observed acceleration is less than that expected from gravity alone. Total observations were 168 per participant. The $p$-values for the $r \mathrm{~s}$ in the All and Positive Torque columns were all $<.001$. None of the negative torque $r$ s was significant; all entries in the last column were significant

\section{Discussion}

Previous research demonstrated that gravitational torque and moment of inertia are relevant to perceiving the locations of the ends of a rod rotated in a vertical plane about an axle (Michaels \& Isenhower, in press). The simplicity of the paradigm and findings made it a promising candidate for testing whether parallels of these variables constituted an information space, and whether a parallel to that information space could be found in exploration space. To do this, we attempted a partial replication of Michaels and Isenhower's Experiment 1 in which we now measured exploration. An additional change was that feedback was given on the middle two blocks of a four-block series, to see whether information-use changed. It was hypothesized that such initial attunements in variable space, as well as any reattunement, would be manifested in exploration.

As had been shown by Michaels and Isenhower (in press), perceivers again succeeded in reporting the partial lengths, and all but one, who had started with the highest judgment-length correlation, showed some improvement. Improvement appeared to entail both decreases in variability (Gibson \& Bergman, 1954; Wagman, Shockley, Riley, \& Turvey, 2001) and, with some perceivers, reattunement to a better locus in variable space (Michaels et al., 2008), nearing the ratio of the weighting of moment of inertia to the weighting of gravitational torque at which partial length was specified. Importantly, with some perceivers the ratio started below the optimal and increased; with another it started above the optimal and decreased.

The relevant physics had two important implications. First, the similarity between the regression equation and Equation 1 implied that $T_{m}$ could be understood as an information variable. Second, Equation 1 implicated two characteristics of exploration that could determine the locus in that information space: the angular acceleration of the rod and the angle at which the torque creating that acceleration was applied. The first of these proved the more important; the results revealed notable similarities between the locus as determined from regressions of judgments against $I$ and $T_{g}$ (as shown in Fig. 4), and average peak acceleration (as shown in Fig. 6). Although details were not given in the results section, we did not find a rod-orientation variable that explained significant additional variance. It was concluded that the perceivers' positions on the $I-T_{g}$ information line on blocks of trials and movement over blocks were related to the rotational acceleration of the rods. More specifically, the results suggested that perceivers try to accelerate the rod a fixed amount, and the $T_{m}$ that is required informs the perceived partial length. Reattunement to a new locus in information space is achieved by changing the attempted acceleration.

As noted, the rough correspondence between locus on the information line and acceleration held both between and within individuals. We expressed disappointment that there was not more change within participants; only two participants had large movements in information space that could be (and were) paralleled by movements in action space. As to why there was not the degree of reattunement that had been found in earlier studies (Arzamarski et al., 2010; Michaels et al., 2008), we can only speculate. One possibility is that asking perceivers to make two judgments on each trial-of the left extent and the right extent - and giving feedback on both might have been too difficult for some participants. Dynamic touch studies that have shown more reattunement were single-judgment-plus-feedback trials on which the tobe-perceived object was held at one end. ${ }^{5}$

\footnotetext{
${ }^{5}$ A reviewer expressed concern that asking participants to make two judgments might encourage perception of the length of the whole rod and of the position of the hand relative to the whole length, from which the two partial length judgments could be derived. To provide a crude test of whether this happened, we compared the correlations of the perceived and actual partial lengths (left-left and right-right) with the correlations between actual and perceived whole length (i.e., the sum of the partial lengths), and actual and perceived hand position. The average partial- and whole-length correlations were not statistically different (.78 vs. .75), but both were higher than the position correlation $(.59), F(2,10)=7.59, p=.0372$, with the Hunyh-Feldt correction. One would expect that partial-length correlations would not be higher than position correlations if partial-length judgments were derived in part from perceived hand position.
} 
The key role of an action parameter, angular acceleration, in determining detected information was further supported by significant correlations between perceived partial length and the muscle torque needed to accelerate the rod in the observed direction and amount. Together the symmetry of the locus on the information line and observed acceleration, and the success of predicting judgment on the basis of exerted muscle torque led to the tentative conclusion that the information line and the action line are two sides of the same coin.

A corollary of the information-action space dual is its implication for cue combination. This issue is critical to the theories of direct perception and direct learning, the former because of multiple specification, and the latter because its use of lower order variables to create variable spaces invites the speculation that learning involves cognitive cuereweighting. In the present case, the information line was constructed from two variables, $I$ and $T_{g}$, and the apparent demonstration that the exploited locus on the line was a function of how the rod was manipulated presents an alternative to a cue-combination account of variable use in dynamic touch. Acceleration weights $I$ relative to $T_{g}$, and therefore locates the perceiver in information space. $T_{m}$ is indeed a weighted sum, but it can come in as a weighted sum based on acceleration, and not as detected parts originating in holding vs. accelerating, and combined cognitively.

A related conclusion was recently drawn by Arzamarski (2009). Arzamarski asked perceivers to report the length or width of pipes supported on two axes, which permitted independent rotations in a vertical plane and about the longitudinal axis of the pipe. Rotations in the vertical plane were resisted by the first principal moment of inertia, $I_{1}$, and rotations on the longitudinal axis were resisted by the third principal moment, $I_{3}$. Good performance required the contribution of both variables, but participants were unable to combine the two independently available variables. Arzamarski concluded that the "combination" of the two variables was only observed with unrestricted wielding, which we might now understand as when wielders could functionally weight the variables with exploration.

An enumeration of elementary cues (e.g., $I$ or $T_{g}$ ) might seem like an innocent starting point for an informational ontology. Similarly, the success of those cues in predicting judgments in multiple regression analyses might seem to confirm their rightful place as inputs for combinatorial algorithms. An exploration-based view, on the other hand, suggests that their status as components might be a fiction, rooted in the arbitrary character of the metrics adopted in the first place. Imagine that a physicist were to use a coordinate system indifferent to the direction of gravity. If it was further concluded that different forces acted along the three axes, and that they combined to accelerate objects toward the earth, we would all recognize the three forces as fictions and their combination as unnecessary. Again, this general point reflects a recurring theme of the ecological approach. From ecological optics to affordances (Gibson, 1979/1986), formulating an ontology appropriate for perceiving and acting creatures - $\mathrm{a}$ non-elementaristic ontology - is an indispensable scientific goal (see also Jacobs \& Michaels, 2007; Runeson, 1977, 1994).

The idea that inertial and gravitational moments can differentially affect judgments under different circumstances is one that has been promoted elsewhere. Burton and Turvey (1990) also showed that constraints on wielding ("do not move the rod") favored use of static moment (which in their Experiment was perfectly correlated with $T_{g}$ ), whereas freewielding favored the use of $I$. It was suggested by van de Langenberg, Kingma, and Beek $(2006,2008)$ that whether perceivers rely on moment of inertia or static moment depends on the salience of the variables. They defined salience in terms of signal-to-noise ratios of individual variables, such as mass, static moment, moment of inertia, and period of oscillation. For example, if a collection of objects whose length is to be judged is contrived to have equal moments of inertia, but different static moments, judgments will correlate more highly with static moment (Kingma, Beek, \& van Dieën, 2002). If static moment is held constant, and moment of inertia varied, judgments will correlate more highly with moment of inertia (Kingma, van de Langenberg, \& Beek, 2004). Similarly, if a rod is held horizontally, static moment will be more salient than if it is held vertically (van de Langenberg et al., 2006; see also Harrison, Hajnal, Lopresti-Goodman, Isenhower, \& Kinsella-Shaw, in press). Moreover, van de Langenberg et al. (2006) mention exploration as a factor affecting salience. This last point, especially, is close to the position presented here. Their study of the effects of exploration on variable use concentrated on the experimenter's manipulation of variable use, whereas our emphasis is on the wielders' discovering the best exploration. Nevertheless, van de Langenberg et al. (2006) expressed sensitivity to the learning aspects of exploration when they suggested the possible fruitfulness of looking at differences in exploration between novices and experts. A difference, however, between the current position and that of Burton and Turvey and van de Langenberg et al. (2006) is that they tend to emphasize a two-variable conception of information, which revives the cuecombination issue, as well as its ecological variants (e.g., directed perception, Cutting, 1986). Their multiple variables also were dimensionally different object properties $\left(\mathrm{kg}, \mathrm{kg}-\mathrm{m}^{2}\right.$, time, etc.; see also Jacobs et al., 2009). Here we have emphasized a common currency, a single detectable variable, $T_{m}$, that could inform length perception under all circumstances; its value depends on gravity, orientation, intended acceleration, and moment of inertia. 
We conclude with two issues regarding the generality of the findings reported here. The first concerns whether these findings from partial length judgments of a rod rotated on an axle generalize to free wielding and to whole-length perception. The second concerns whether the conclusion that information space is a dual of exploration space is generalizable to other perceptual systems.

Three characteristics distinguish the current paradigm from more typical dynamic touch studies. One is the partial-length aspect. Usually perceivers asked to report length are to report entire length, and most often while the rod is held at one end. We expect that the relation described here, that judgments of length are a function of the sum of gravitational and inertial torques, holds in both cases. Grasping a rod at one end is just a special case of partial length perception, with one partial length being near zero. That said, if the instruction is to report only one side, wielding might be different: A wielder might not try to accelerate equally in the two directions; accelerating the distal end of the rod upward would provide a sufficient informational basis for judgment. A wielder might simply let the rod drop from gravity alone before the next information-gathering upstroke. There was a penalty for doing this in the present experiment (see Table 3), but it would not affect performance for a rod held at the end. Relatedly, if one were asked to report the entire length of a rod held along its length, accelerative rotations would be required in both directions.

As to whether rotating a rod on an axle severely limits the generalizability of the findings, we believe it does not. Often, experimental participants do rotate the object about another fixed axis - in the wrist. The mechanics of wielding a rod in a vertical anterior-posterior plane can be almost identical to that presented here; the only difference is that the hand must support the weight of the rod. If, on the other hand, rotation occurs on more than one axis or the center of mass and rotation point are not coplanar during rotation, exploratory torques create acceleratory components in other planes. While the dynamical equations are more complicated, judgments could still be a function of muscle-based torque.

The final question is whether the general principle proposed here - that exploration directly accesses information loci without recourse to underlying variables - is an appropriate characterization of perception and perceptual learning in general. Or is it unique to dynamic touch, which can so obviously depend on active transformations to reveal invariant properties? Already mentioned was Drewing and Ernst's (2006) research, which demonstrated that the vertical force that a perceiver exerts in exploratory rubbing of a surface differentially weights cues in haptic shape perception. Kaim and Drewing (2010) went on to demonstrate that an exploration-based integration model accounted for results that could not be accounted for by cognitive integration of individual cues using a maximum likelihood estimation model (e.g., Ernst \& Banks, 2002).

An even broader generalization might be sought via the link between exploratory and performatory actions. Performatory movements also entail transformations that make available variants and invariants. One understanding of the basis of successful action is that an actor must move so as to create a particular invariant. As examples, achieving a tau-dot of -.5 would lead to successful braking (Lee, 1976); achieving a vertical optical acceleration of 0 of the image of a ball on an image plane would lead to a successful fly-ball interception (Chapman, 1968). In short, acting appropriately so as to create certain information (or learning how to do so) does not obviously distinguish between exploring and performing, the perceiver's intention aside. An appropriate manner of performing can put one at some locus in information space, as demonstrated by Smith, Flach, Dittman, and Stanard (2001). In sum, we see no obvious limitations of the generality of the thesis that information variables are accessed directly through action, and its corollary — that cues are not combined.

\section{References}

Arzamarski, R. (2009). Effects of exploration on learning and attunement in dynamic touch (Unpublished doctoral dissertation). University of Connecticut.

Arzamarski, R., Isenhower, R. W., Kay, B. A., Turvey, M. T., \& Michaels, C. F. (2010). Effects of intention and learning on attention to information in dynamic touch. Attention, Perception, \& Psychophysics, 72, 721-735.

Brunswik, E. (1952). The conceptual framework of psychology. Oxford: University of Chicago Press.

Burton, G., \& Turvey, M. T. (1990). Perceiving the lengths of rods that are held but not wielded. Ecological Psychology, 2, 295324.

Carello, C., Santana, M.-V., \& Burton, G. (1996). Selective perception by dynamic touch. Perception \& Psychophysics, 58, 1177-1190.

Chapman, S. (1968). Catching a baseball. American Journal of Physics, 36, 868-870.

Cutting, J. E. (1986). Perception with an eye for motion. Cambridge: MIT.

Drewing, K., \& Ernst, M. O. (2006). Integration of force and position cues for shape perception through active touch. Brain Research, 1078, 92-100.

Ernst, M. O., \& Banks, M. S. (2002). Humans integrate visual and haptic Information in a statistically optimal way. Nature, 415, 429-433.

Gibson, J. J. (1966). The senses considered as perceptual systems. Boston: Houghton Mifflin.

Gibson, J. J. (1979/1986). The ecological approach to visual perception. Boston, MA: Houghton Mifflin.

Gibson, E. J., \& Bergman, R. (1954). The effect of training on absolute estimation of distance over the ground. Journal of Experimental Psychology, 48, 473-480.

Harrison, S. J., Hajnal, A., Lopresti-Goodman, S., Isenhower, R. W., \& Kinsella-Shaw, J. M., (in press). Perceiving action-relevant 
properties of tools through dynamic touch: Effects of mass distribution, exploration style, and intention. Journal of Experimental Psychology: Human Perception and Performance.

Jacobs, D. M., \& Michaels, C. F. (2007). Direct learning. Ecological Psychology, 19, 321-349.

Jacobs, D. M., Silva, P. L., \& Calvo, J. (2009). An empirical illustration and formalization of the theory of direct learning: The muscle-based perception of kinetic properties. Ecological Psychology, 21, 245289.

Kaim, L., \& Drewing, K. (2010). Exploratory pressure influences haptic shape perception via force signals. Attention, Perception, \& Psychophysics, 72, 823-838.

Kingma, I., Beek, P. J., \& van Dieën, J. H. (2002). The inertia tensor versus static moment and mass in perceiving length and heaviness of hand-wielded rods. Journal of Experimental Psychology: Human Perception and Performance, 28, 180-191.

Kingma, I., van de Langenberg, R., \& Beek, P. J. (2004). Which mechanical invariants are associated with the perception of length and heaviness of a nonvisible handheld rod? Testing the inertia tensor hypothesis. Journal of Experimental Psychology: Human Perception and Performance, 30, 346-354.

Lederman, S. J., \& Klatzky, R. L. (1987). Hand movements: A window into haptic object recognition. Cognitive Psychology, 19, 342-368.

Lederman, S. J., \& Klatzky, R. L. (1990). Haptic classification of common objects: Knowledge-driven exploration. Cognitive Psychology, 22, 421-459.

Lee, D. N. (1976). A theory of visual control of braking based on information about time-to-collision. Perception, 5, 437-459.

Michaels, C. F., \& Isenhower, R. W. (in press). An information space for partial length perception in dynamic touch, Ecological Psychology.
Michaels, C. F., Arzamarski, R., Isenhower, R. W., \& Jacobs, D. M. (2008). Direct learning in dynamic touch. Journal of Experimental Psychology: Human Perception and Performance, 34, 944-957.

Riley, M. A., Wagman, J. B., Santana, M., Carello, C., \& Turvey, M. T. (2002). Perceptual behavior: Recurrence analysis of a haptic exploratory procedure. Perception, 31, 481-510.

Runeson, S. (1977). On the possibility of 'smart' perceptual mechanisms. Scandinavian Journal of Psychology, 18, 172-179.

Runeson, S. (1994). Psychophysics: The failure of an elementaristic dream. The Behavioral and Brain Sciences, 17, 761-763.

Smith, M. R., Flach, J. M., Dittman, S. M., \& Stanard, T. (2001). Monocular optical constraints on collision control. Journal of Experimental Psychology: Human Perception and Performance, 27, 395-410.

Turvey, M. T., Carello, C., \& Kim, N.-G. (1990). Links between active perception and the control of action. In H. Haken \& M. Stadler (Eds.), Synergetics of cognition (pp. 269-295). Heidelberg: Springer.

van de Langenberg, R., Kingma, I., \& Beek, P. J. (2006). Mechanical invariants are implicated in dynamic touch as a function of their salience in the stimulus flow. Journal of Experimental Psychology: Human Perception and Performance, 32, 1093-1106.

van de Langenberg, R., Kingma, I., \& Beek, P. J. (2008). The perception of limb orientation depends on the center of mass. Journal of Experimental Psychology: Human Perception and Performance, 34, 624-639.

Wagman, J. B., Shockley, K., Riley, M. A., \& Turvey, M. T. (2001). Attunement, calibration, and exploration in fast haptic perceptual learning. Journal of Motor Behavior, 33, 323-327.

Yuille, A. L., \& Bülthoff, H. H. (1996). Bayesian decision theory and psychophysics. In D. C. Knill \& W. Richards (Eds.), Perception as Bayesian inference (pp. 123-161). Cambridge: Cambridge University Press. 